\title{
Diastolic dysfunction in a pre-clinical model of diabetes is associated with changes in the cardiac non-myocyte cellular composition
}

Charles D. Cohen ${ }^{1,2,5}$, Miles J. De Blasio', Man K. S. Lee ${ }^{3}$, Gabriella E. Farrugia², Darnel Prakoso', Crisdion Krstevski ${ }^{2,5}$, Minh Deo ${ }^{1}$, Daniel G. Donner ${ }^{4,6}$, Helen Kiriazis ${ }^{4,6}$, Michelle C. Flynn', Taylah L. Gaynor ${ }^{2,5}$, Andrew J. Murphy ${ }^{3}$, Grant R. Drummond ${ }^{5}$, Alexander R. Pinto ${ }^{2,5^{*}+}$ and Rebecca H. Ritchie ${ }^{1,5^{*+}}$ (D)

\begin{abstract}
Background: Diabetes is associated with a significantly elevated risk of cardiovascular disease and its specific pathophysiology remains unclear. Recent studies have changed our understanding of cardiac cellularity, with cellular changes accompanying diabetes yet to be examined in detail. This study aims to characterise the changes in the cardiac cellular landscape in murine diabetes to identify potential cellular protagonists in the diabetic heart.

Methods: Diabetes was induced in male FVB/N mice by low-dose streptozotocin and a high-fat diet for 26-weeks. Cardiac function was measured by echocardiography at endpoint. Flow cytometry was performed on cardiac ventricles as well as blood, spleen, and bone-marrow at endpoint from non-diabetic and diabetic mice. To validate flow cytometry results, immunofluorescence staining was conducted on left-ventricles of age-matched mice.

Results: Mice with diabetes exhibited hyperglycaemia and impaired glucose tolerance at endpoint. Echocardiography revealed reduced E:A and e':a' ratios in diabetic mice indicating diastolic dysfunction. Systolic function was not different between the experimental groups. Detailed examination of cardiac cellularity found resident mesenchymal cells (RMCs) were elevated as a result of diabetes, due to a marked increase in cardiac fibroblasts, while smooth muscle cells were reduced in proportion. Moreover, we found increased levels of Ly $6 C^{\text {hi }}$ monocytes in both the heart and in the blood. Consistent with this, the proportion of bone-marrow haematopoietic stem cells were increased in diabetic mice.

Conclusions: Murine diabetes results in distinct changes in cardiac cellularity. These changes-in particular increased levels of fibroblasts - offer a framework for understanding how cardiac cellularity changes in diabetes. The results also point to new cellular mechanisms in this context, which may further aid in development of pharmacotherapies to allay the progression of cardiomyopathy associated with diabetes.
\end{abstract}

Keywords: Cardiac cellularity, Diabetes, Flow cytometry, Echocardiography, Fibroblast

\footnotetext{
*Correspondence: alex.pinto@baker.edu.au; Rebecca.ritchie@monash.edu ${ }^{\dagger}$ Alexander R. Pinto and Rebecca H. Ritchie Equal contribution

1 Heart Failure Pharmacology, Drug Discovery Biology, Monash Institute of Pharmaceutical Sciences, 399 Royal Parade, Parkville, VIC 3052, Australia

${ }^{2}$ Cardiac Cellular Systems, Baker Heart and Diabetes Institute, 75 Commercial Road, Melbourne, VIC 3004, Australia

Full list of author information is available at the end of the article
}

\section{Background}

Diabetes mellitus is a leading cause of death worldwide, with a total global prevalence exceeding 450 million individuals [1]. In 2015, diabetes was attributed to $12.8 \%$ of total all-cause mortality worldwide, providing a substantial socioeconomic burden and health concern $[2,3]$.

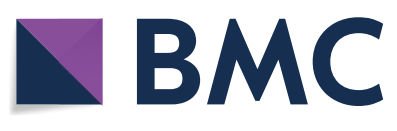

(c) The Author(s) 2021. This article is licensed under a Creative Commons Attribution 4.0 International License, which permits use, sharing, adaptation, distribution and reproduction in any medium or format, as long as you give appropriate credit to the original author(s) and the source, provide a link to the Creative Commons licence, and indicate if changes were made. The images or other third party material in this article are included in the article's Creative Commons licence, unless indicated otherwise in a credit line to the material. If material is not included in the article's Creative Commons licence and your intended use is not permitted by statutory regulation or exceeds the permitted use, you will need to obtain permission directly from the copyright holder. To view a copy of this licence, visit http://creativeco mmons.org/licenses/by/4.0/. The Creative Commons Public Domain Dedication waiver (http://creativecommons.org/publicdomain/ zero/1.0/) applies to the data made available in this article, unless otherwise stated in a credit line to the data. 
Diabetes is associated with a significantly elevated risk of cardiovascular death and hospitalisation for heart failure (HF) $[4,5]$. However, there remains no specific treatment for HF or its development in individuals with diabetes. HF in diabetes is often accompanied by impaired cardiac output, cardiac fibrosis, cardiomyocyte hypertrophy, cell death, and oxidative stress [6]. Diabetes also involves chronic and systemic inflammation [7, 8] with monocytosis and neutrophilia [7-9]. Despite extensive efforts to characterise diabetes-induced HF, inherent cellular mechanisms underpinning cardiac dysfunction in diabetes remain to be ascertained.

The mammalian heart consists of a diverse range of cell types [10]. Cardiac non-myocytes-comprised of endothelial cells (ECs), resident mesenchymal cells (RMCs) and leukocytes-outnumber myocytes, and are critical for maintaining homeostasis of the heart $[10,11]$. While a number of recent studies have provided valuable new insights into the disparate roles of non-myocyte cells in cardiac homeostasis $[10,12,13]$ and pathological remodelling [14-16], the cellular dynamics of nonmyocytes during development of diabetes-induced heart failure remains unexplored. Using a recently published murine model of diabetes-induced cardiomyopathy [17], this study aimed to determine the difference in cardiac non-myocyte cellular proportions compared to nondiabetic mice. Here, we show that experimental diabetes impacts multiple cellular compartments in the heart, providing a framework for understanding the cellular dynamics and mechanisms driving development of diabetes-induced heart failure.

\section{Research design and methods Animal experiments}

All animal-related experiments were approved by the Alfred Research Alliance (ARA) Animal Ethics Committee (Ethics number: E/1681/2016/B) and were performed in accordance with the National Health and Medical Research Council of Australia. FVB/N mice were sourced from the ARA Animal Services (provided in three separate cohorts). Mice had access to food and water ad libi-

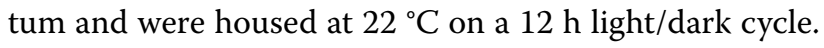
Male 6-week-old FVB/N mice were randomly allocated into the non-diabetic $(\mathrm{ND}, \mathrm{n}=7)$ citrate vehicle control group fed standard chow diet, or diabetes mellitus (diabetes, $\mathrm{n}=19$ ) which was induced by the combination of low-dose streptozotocin (STZ; cat\# AG-CN2-0046, AdipoGen Life Sciences, NSW, Australia) and high-fat-diet (HFD; SF04-001, Specialty Feeds, WA, Australia, 43\% total calculated digestible energy from lipids). STZ was administered by three consecutive daily intraperitoneal (i.p.) injections $(55 \mathrm{mg} / \mathrm{kg}$ body weight in $0.1 \mathrm{~mol} / \mathrm{L} \mathrm{cit-}$ ric acid vehicle, $\mathrm{pH} 4.5$ [cat\# 251275, Sigma-Aldrich,
USA]). Mice administered STZ were subsequently fed a HFD ad libitum for 26-weeks, as previously described [17]. Blood glucose levels were measured fortnightly via saphenous vein bleeds using a glucometer (Accu-Chek ${ }^{\circledR}$ Performa II, Roche Diagnostics, NSW, Australia). Intraperitoneal glucose and insulin tolerance tests were conducted at endpoint (26-weeks of diabetes) to assess glucose clearance and insulin resistance, as previously described [17]. Whole-body composition analysis was performed at endpoint using an Echo-MRI ${ }^{\mathrm{TM}}$ 4-in-1 700 Analyser (EchoMRI, Houston, TX, USA) to assess percentage fat mass and total lean mass. Percentage glycated haemoglobin $\left(\% \mathrm{HbA}_{1 \mathrm{c}}\right)$ was also measured at endpoint to assess long-term blood glucose levels (Cobas b 101 POC system, Roche Diagnostics, NSW, Australia). Mice were euthanised by administration of Ketamine/Xylazine $(85 / 8.5 \mathrm{mg} / \mathrm{kg}$, i.p.) and subsequent cardiac exsanguination. As previously described [10-12], the thoracic cavity was exposed and right atrium was cut to allow for cardiac perfusion through the left-ventricular apex (PBS, $0.9 \mathrm{mM}$ $\mathrm{CaCl}_{2}, 200 \mathrm{mM} \mathrm{KCl}$ ), after which the heart was excised and ventricles were used for flow cytometry.

\section{Echocardiography}

Echocardiography was conducted in mice under anaesthesia (Ketamine/Xylazine/Atropine [KXA], $80 / 8 / 0.96 \mathrm{mg} / \mathrm{kg}$, i.p.) at 26 -weeks post diabetes (32weeks of age) using a Philips iE33 ultrasound machine with a $15-\mathrm{MHz}$ linear-array transducer. Analysis was conducted at the Baker Heart and Diabetes Institute and quality control was completed by technicians at the Preclinical Cardiology Microsurgery \& Imaging Platform (PCMIP). Doppler flow echocardiography was used to assess cardiac transmitral flow velocity in each phase of diastole, where the early phase (E wave) and the late phase (A wave) were measured to determine the E:A ratio. Similarly, tissue Doppler was performed to examine the tissue motion of the mitral annulus (early phase $=\mathrm{e}$, late phase $=a^{\prime}$ wave). M-mode echocardiography was conducted to assess left ventricle (LV) systolic function. Variables obtained from M-mode analysis included LV end-diastolic dimension (LVEDD) and LV end-systolic dimension (LVESD) to calculate fractional shortening $(\% \mathrm{FS}=[(\mathrm{LVEDD}-\mathrm{LVESD}) / \mathrm{LVEDD}] \times 100)$.

\section{Flow cytometry \\ Blood, spleen and bone marrow}

Whole blood was obtained by cardiac puncture at endpoint and stained using a leukocyte-specific antibody panel (Additional file 1: Table S4). Bone marrow from the tibia and femur were flushed using PBS without $\mathrm{Mg}^{2+}$ and $\mathrm{Ca}^{2+}$ into $50 \mathrm{~mL}$ centrifuge tubes. Spleens were manually dissociated and passed through a $35 \mu \mathrm{M}$ 
filter into $50 \mathrm{~mL}$ centrifuge tubes to obtain a single cell suspension as previously described [7]. Blood, spleen and bone marrow were then subjected to red blood cell (RBC) lysis for $15 \mathrm{~min}$ at $4{ }^{\circ} \mathrm{C}$ using an ammonium chloride based commercial lysis buffer (1X dilution, 555899, Becton Dickinson, USA). After RBC lysis, the remaining stained cells were washed twice in 'Fx buffer' ( $1 \mathrm{X}$ HBSS [Gibco ${ }^{\mathrm{TM}}$, NY, USA], 2\% FCS). Between each wash, cells were centrifuged at $400 \times g$ for $5 \mathrm{~min}$ at $4{ }^{\circ} \mathrm{C}$. Cells were then resuspended in $200 \mu \mathrm{l}$ of Fx buffer containing 4',6-diamidino-2-phenylindole (DAPI $[0.1 \mu \mathrm{g} / \mathrm{mL}]$ ); and filtered through $35 \mu \mathrm{M}$ mesh into $5 \mathrm{ml}$ polystyrene round-bottom tubes (352052, Falcon ${ }^{\circledR}$, NY, USA) for flow cytometry. Gating strategies for each of the above cell suspensions are provided in Additional file 1: Figures S3S5. For normalisation of flow cytometry data, $20 \mu \mathrm{l}$ of blood was used to measure total white blood cell count using a Sysmex XS-1000i Hematology Analyzer.

\section{Heart}

High-dimensional flow-cytometry was performed on cardiac ventricles (comprising the $\mathrm{LV}$, ventricular septum and right ventricle) from ND and mice with diabetes. Following perfusion, hearts were minced using curved scissors (14077-09, Walton, USA) as previously described [10], and transferred to $5 \mathrm{ml}$ microfuge tubes for enzymatic digestion at $37{ }^{\circ} \mathrm{C}(2 \mathrm{mg} / \mathrm{mL}$ collagenase type IV [LS004188, Worthington Biochem, NJ, USA], $1 \mathrm{mg} /$ $\mathrm{mL}$ Dispase II [04942078001, Roche, NSW, Australia] in $0.9 \mathrm{mM} \mathrm{CaCl}_{2}$ in PBS). Cardiac non-myocyte cells were triturated three times at 15 -min intervals using a Pasteur pipette to mechanically aid enzymatic digestion for a total of $45 \mathrm{~min}$. Digested non-myocyte cardiac cells were then filtered through $75 \mu \mathrm{M}$ nylon mesh into a $15 \mathrm{~mL}$ tube containing $10 \mathrm{~mL}$ of cold PBS $\left(0.9 \mathrm{mM} \mathrm{CaCl}_{2}\right)$ and subjected to centrifugation $\left(200 \mathrm{~g}, 15 \mathrm{~min}, 4{ }^{\circ} \mathrm{C}-\right.$ no breaks) for debris clearance. The majority of the supernatant was aspirated and the remaining volume $(\sim 1 \mathrm{~mL})$ was washed with a further $1 \mathrm{~mL}$ of Fx buffer supplemented with $0.9 \mathrm{mM} \mathrm{CaCl} 2$. Cells were pelleted at $400 \times g$ $\left(4 \mathrm{~min}, 4^{\circ} \mathrm{C}\right.$ ) and resuspended in $200 \mu \mathrm{l}$ of Fx Buffer with $\mathrm{Ca}^{2+}$ to yield the single cell suspension of non-myocyte cardiac cells. Cells were then stained using the antibody panel designed for examining the non-myocyte fraction of the heart (Additional file 1: Table S5). Cells were strained through a $35 \mu \mathrm{m}$ filter and flow cytometry was performed on a BD LSR Fortessa ${ }^{\mathrm{TM}} \mathrm{X}-20$ Special Order system located at the Baker Heart and Diabetes Institute.

\section{Histological analysis}

Age and sex-matched, fresh-frozen LV samples embedded in Optimal Cutting Temperature (OCT) compound were acquired from a separate cohort of ND and mice with diabetes [17] for histological analysis. LV sections were cut $(10 \mu \mathrm{m})$ on a cryostat (CM1950, Leica Biosystems) for staining (ND: $n=11$, diabetes: $n=11)$. LV sections were co-stained with GATA4 (1:100, 14-9980-80, eBioScience $^{\mathrm{TM}}$, Invitrogen, Australia) and PCM1 (1:100, 19856-1-AP, ProteinTech Group, USA) antibodies to delineate the cell abundance of RMCs $\left(\mathrm{PCM}^{-}{ }^{-} \mathrm{GATA}^{+}\right.$ cells) as recently reported [12]. Serial sections were stained with DACH1 (1:100, 10914-1-AP, ProteinTech, USA) to quantify EC abundance [10, 12]. All immunofluorescence sections were counterstained with DAPI to identify total cell nuclei. Immunofluorescence micrographs of each LV sample were acquired at a 20X objective and tiled $(3 \times 3$ fields of view $)$ on a Nikon A1R confocal laser scanning microscope. Quantified values of immunofluorescence signal were normalised to total nuclei $\left(\mathrm{DAPI}^{+}\right)$.

\section{Statistical analysis}

Flow cytometry data was analysed using FlowJo (v10.7.1) software. Raw cardiac flow cytometry data was normalised to the mean of the ND values within each batch, such that the mean of each ND cell type is equal to 1 . Raw blood flow cytometry data was normalised to total whiteblood cell count obtained from the hematology analyser, then subsequently batch normalised as aforementioned. Immunofluorescence micrographs were analysed by QuPath software (v0.2.3), using the cell count function to quantify nuclei. Echocardiography data was analysed using RadiAnt DICOM viewer software (v2020.2), after which quantification was performed in accordance with the PCMIP guidelines. All data was illustrated and analysed statistically using GraphPad Prism (v8.1.2). Comparison of experimental groups was conducted using an unpaired $t$-test, whereby statistical significance was determined as $P<0.05$.

\section{Results \\ The STZ-HFD model recapitulates primary features of diabetes}

The presence of diabetes was confirmed by a range of physiological tests prior to euthanasia. Consistent with our previous report [17], mice with diabetes exhibited significantly elevated blood glucose at endpoint (Additional file 1: Table S1). This was corroborated by measurement of glycated haemoglobin $\left(\% \mathrm{HbA}_{1 \mathrm{c}}\right)$ at endpoint, which was significantly increased in mice with diabetes $(P<0.0001$; Additional file 1: Table S1). In this study however, mice exhibiting diabetes did not gain more weight than their ND counterparts (Additional file 1: Table S1). This was recapitulated by the EchoMRI body composition analysis, showing no differences in lean or fat mass (Additional file 1: Table S1) between experimental 
groups. Impaired glucose tolerance was evident in mice with diabetes, indicating reduced clearing efficiency of systemic glucose, presented as the area under the curve (AUC, $P<0.0001$, Additional file 1: Table S1). In contrast, there was no difference in the AUC from the insulin tolerance test between ND and mice with diabetes (Additional file 1: Table S1).

\section{STZ-HFD mice exhibit LV diastolic dysfunction, but not systolic dysfunction}

Echocardiography measurements of LV diastolic and systolic function were recorded in vivo, to determine the degree of cardiac functional impairment in mice with diabetes relative to their ND counterparts. Pulsed-wave Doppler echocardiography was conducted to measure mitral blood flow velocity during the early (E-wave) and late (A-wave) filling phases of diastole (Additional file 1: Fig. S1A). Heart rate (HR) tended to be elevated in mice with diabetes, but this did not reach statistical significance $(P=0.07$; Additional file 1: Figure S1B). Although no differences were detected in the peak $\mathrm{E}$ wave (Additional file 1: Figure S1C), the peak A wave velocity was significantly elevated in mice with diabetes compared to ND mice $(P<0.05$; Additional file 1: Figure S1D). Consequently, a significant reduction in E:A ratio (a hallmark feature of diastolic dysfunction) was observed in diabetic hearts vs. ND $(P<0.05$ Additional file 1: Figure S1E). There were no differences in other measurements of diastolic function including deceleration time or isovolumic relaxation time (IVRT) between experimental groups (Additional file 1: Figure S1F, G, respectively).

To accompany transmitral blood flow, tissue Doppler echocardiography was used to assess the velocity of the mitral valve itself in each phase of diastole $\left(e^{\prime}=\right.$ early phase, a' late phase, Additional file 1: Figure $\mathrm{S} 1 \mathrm{H}-\mathrm{L}$ ). Although the peak e' velocity was only modestly reduced ( $P=0.054$, Additional file 1: Figure S1I) and the peak a' velocity exhibited a minor increase $(P=0.072$, Additional file 1: Figure S1J), the e':a' ratio was significantly lower in mice with diabetes compared to ND mice $(P<0.05$, Additional file 1: Figure S1K). There were no detectable changes in the E:e' ratio between cohorts (Additional file 1: Figure S1L).

M-Mode echocardiography was also performed to assess the difference in ventricular wall thickness and systolic function in mice with diabetes. The anterior wall thickness at diastole (AWd), LV end-diastolic dimension (LVEDD) and posterior wall thickness at diastole (PWd) were not different between groups (Additional file 1: Table S2). Interestingly, fractional shortening (\% FS) was significantly elevated in mice with diabetes compared with ND mice $(P<0.05$; Additional file 1: Table S2), consistent with a recent report in spontaneously type-1 diabetic (T1D) Akita mice [18]. Importantly however, diastolic dysfunction was observed in the absence of systolic dysfunction.

\section{Diabetes alters the cardiac non-myocyte cellular composition}

To assess differences in cardiac cellularity associated with diabetes-induced HF, we performed flow cytometric analysis of murine cardiac ventricles at study endpoint. Examination of viable single-cells (see Additional file 1: Figure S2A) revealed significant differences in endothelial cell (EC) and resident mesenchymal cell (RMC) proportions (0.26-fold decrease, twofold increase, respectively), indicating that diabetes alters the relative levels of cardiac non-myocyte cells (Fig. 1A, B). Conversely, leukocytes were at similar levels in ND and mice with diabetes (Fig. 1A, B).

Next, we sought to validate the proportional shifts in EC and RMC populations in diabetes observed by flow cytometry, with immunohistochemical analysis (Fig. 1C, D). To achieve this, we stained left ventricular sections of both cohorts with an antibody cocktail of GATA4 and PCM1 (Fig. 1C) or DACH1 (Fig. 1D), which we have previously employed to quantify proportions of RMCs and ECs [12]. These analyses revealed that RMC $\left(\mathrm{PCM}^{-} \mathrm{GATA}^{+}\right)$cell counts were significantly elevated in diabetic heart sections compared to ND counterparts $(P<0.05$, Fig. 1C). Using the same approach for ECs, serial sections stained with DACH1 indicated no differences in EC abundance between experimental groups (Fig. 1D), suggesting that the proportional difference observed by flow cytometry is due to the increased RMCs [10].

Considering the proportion of RMCs were markedly elevated in the diabetic heart, a range of RMC subtypes were investigated from the initial RMC gate (Additional file 1: Fig.S2). Fibroblasts were significantly increased in diabetic hearts compared to ND (2.36-fold, $P<0.0001$, Fig. 2B). In contrast, the proportion of smooth muscle cells (SMCs), were reduced in the diabetic cohort compared to ND controls $(0.27$-fold, $P<0.05$, Fig. $2 \mathrm{~B})$. No major changes were observed in total mural cells, pericyte or Schwann cell populations (Fig. 2B).

While we did not detect any changes in total resident leukocyte proportions in diabetic mouse hearts compared to ND (Fig. 1B), diabetes has been previously associated with cardiac inflammation and systemic monocytosis $[7,19,20]$. To develop an overview of leukocyte diversity and abundance in diabetic hearts, we identified an array of leukocytes including myeloid and lymphoid cell populations and their subsets (Fig. 3A). There were no differences in cardiac leukocyte subsets between cohorts, except Ly6C $\mathrm{C}^{\text {hi }}$ monocytes, which were 
A

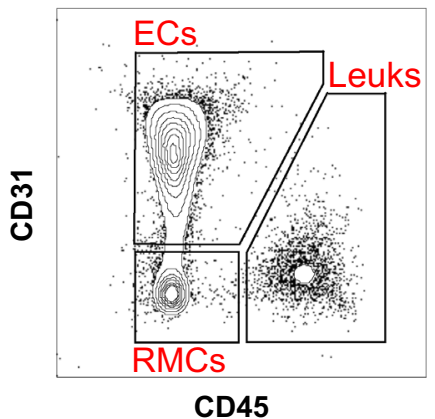

B



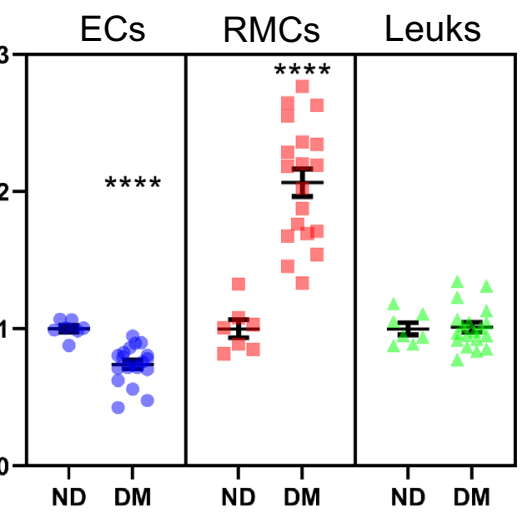

C
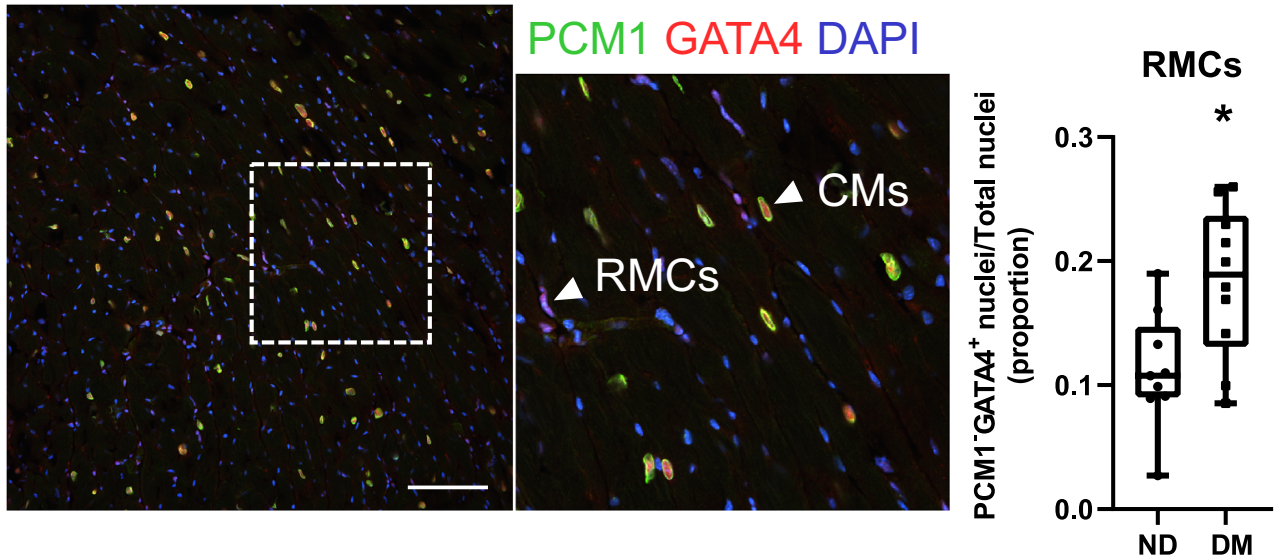

$\mathrm{D}$
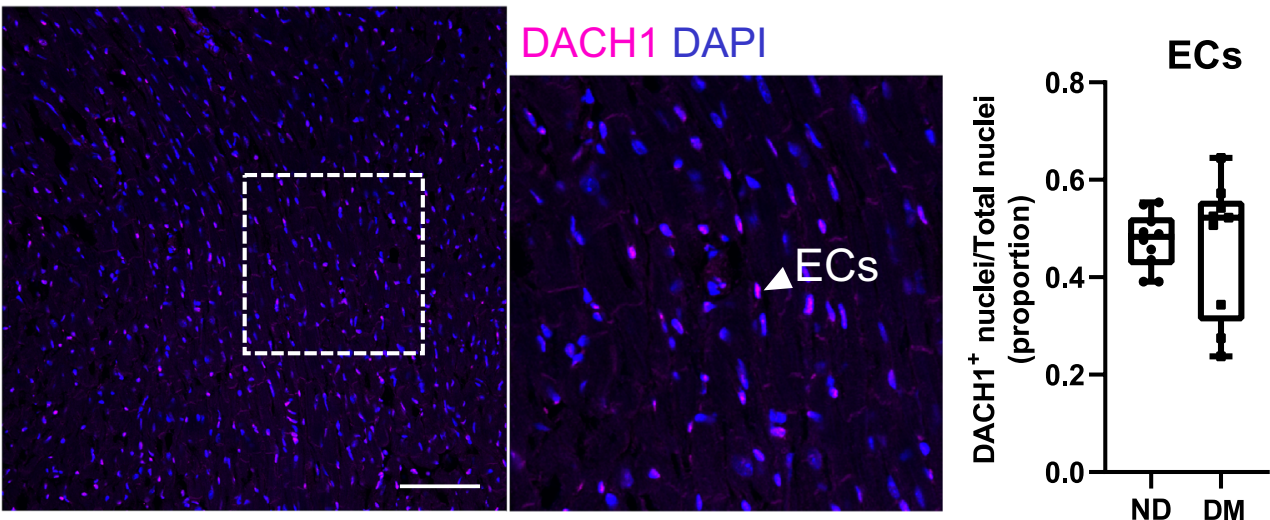

Fig. 1 Differences in the abundance of major non-myocyte cell classes in the diabetic heart. A Flow cytometry contour plot displaying gating of major non-myocyte cell types for quantification of cell type proportion (summarised in B). For full gating strategy see Additional file 1: Figure S2. Endothelial cells $\left(\mathrm{ECs} ; \mathrm{CD}_{1} 1^{+}\right)$, resident mesenchymal cells $\left(\mathrm{RMCs} ; \mathrm{CD} 31^{-} \mathrm{CD} 45^{-}\right)$and leukocytes $\left(\mathrm{Leuks} ; \mathrm{CD}^{+} 5^{+}\right)$. B Proportions of major cell types in non-diabetic $(N D ; n=7)$ and diabetic $(D M ; n=19)$ mouse hearts. Individual sample values are shown with mean $\pm S E M$. $C$ Immunohistochemical analysis of the abundance of RMCs in ND and diabetic mouse heart left ventricles. Left and middle panels show representative confocal micrographs of mouse heart tissue stained for PCM1 and GATA4. PCM1 ${ }^{+} \mathrm{GATA} 4{ }^{+}$and PCM1 ${ }^{-} \mathrm{GATA} 4{ }^{+}$nuclei correspond to nuclei of cardiomyocytes (CM) and RMCs respectively. Nuclei are counterstained with DAPI. Right panel (box-plot) summarises proportion of nuclei corresponding to RMCs in $N D(n=9)$ vs. DM $(n=10)$ enumerated from micrographs. Whiskers of box-and-whisker plot indicate max and min. (D) As for C, heart left ventricle sections were stained with DACH1 to identify nuclei corresponding to endothelial cells in ND $(n=10)$ and $D M(n=9)$ left ventricles. ${ }^{*} P<0.05$, ${ }^{* * * *}$ $P<0.0001$ (Student's unpaired $t$-test). Scale bar $=100 \mu \mathrm{M}$

significantly increased in the myocardium of mice with diabetes (1.8-fold, Fig. 3B). 



Fig. 2 Differences in resident mesenchymal cell (RMC) subtypes in the diabetic heart. A Flow cytometry contour plots display gating strategy for cardiac RMCs and subsets (fibroblasts, SMCs, pericytes and Schwann cells) for quantifying RMC proportions (summarised in B). For full gating strategy see Additional file 1: Figure S2. B Proportions of RMC sub-classes in ND $(n=7)$ and diabetic $(n=19)$ mouse ventricles. Fibro: Fibroblast; Mural: Mural cells; SMCs: smooth muscle cells. Data is displayed as mean \pm SEM. ${ }^{*} P<0.05,{ }^{* * *} P<0.0001$ (Student's unpaired $t$-test)

\section{Circulating Ly6 $\mathrm{C}^{\mathrm{hi}}$ monocytes are elevated in diabetes}

To confirm systemic monocytosis, we quantified circulating leukocytes and their broad subtypes by flow cytometry. As shown previously [7], monocytes, particularly the Ly6C $C^{\text {hi }}$ subset, were significantly elevated in the blood of mice with diabetes (2.2-fold, 2.3-fold, respectively; $P<0.05$ for both; Fig. 4B). Numbers of circulating neutrophils and Ly $_{6} \mathrm{C}^{\text {lo }}$ monocytes were also marginally elevated in diabetic mice compared to their ND counterparts $(P=0.09, P=0.054$ respectively; Fig. $4 \mathrm{~B}$,$) . By con-$ trast, numbers of circulating lymphocytes (B and T-cells) did not differ between cohorts (Fig. 4C).

\section{Systemic monocytosis likely occurs via extramedullary myelopoiesis}

To identify the potential sources of the observed monocytosis in this model, we performed flow cytometry of the bone marrow and spleen. Within the bone marrow, LSKs (haematopoietic stem and progenitor cells; $\left.\left[\mathrm{Lin}^{-} \mathrm{Sca}^{+}{ }^{+} \mathrm{Kit}^{+}\right]\right)$were significantly increased in mice with diabetes (1.8-fold, $P<0.01$, Fig. 5A). However, bone-marrow derived common myeloid progenitors (CMP) and granulocyte-myeloid progenitors (GMP) were not different between experimental groups (Fig. 5A). Monocytes (both $\mathrm{Ly}_{6} \mathrm{C}^{\mathrm{hi}}$ and $\mathrm{Ly}_{6} \mathrm{C}^{\mathrm{lo}}$ ) were significantly increased in the spleen in mice with diabetes compared to their ND controls (1.7-fold, 1.3-fold respectively, $P<0.05$, Fig. 5B). These data suggest that the increased proportion of bone-marrow LSKs could be influencing these cells to mobilise to the spleen to undergo extramedullary myelopoiesis (Fig. 5C).

\section{Discussion}

The relationship between diabetes and HF remains poorly understood. Diabetes-associated cardiac remodellingencompassing myocyte hypertrophy, fibrosis, oxidative stress and apoptosis [21] is well established. However, how the cardiac non-myocyte networks change in diabetes and contribute to this remodelling is unclear. Using a recently characterised mouse model of diabetes-induced cardiomyopathy [17], we aimed to determine how diabetes affects cardiac non-myocyte cell proportions and 

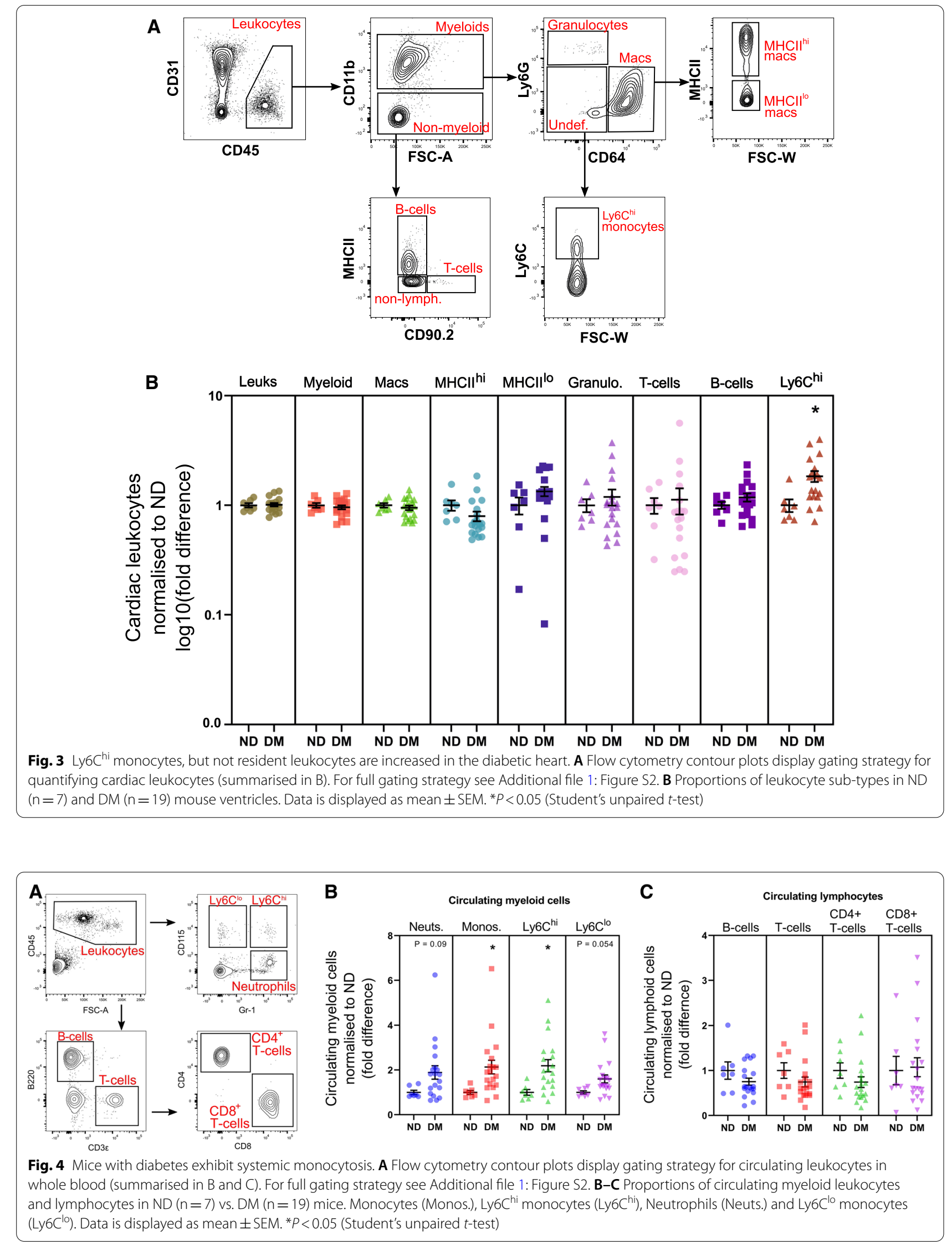

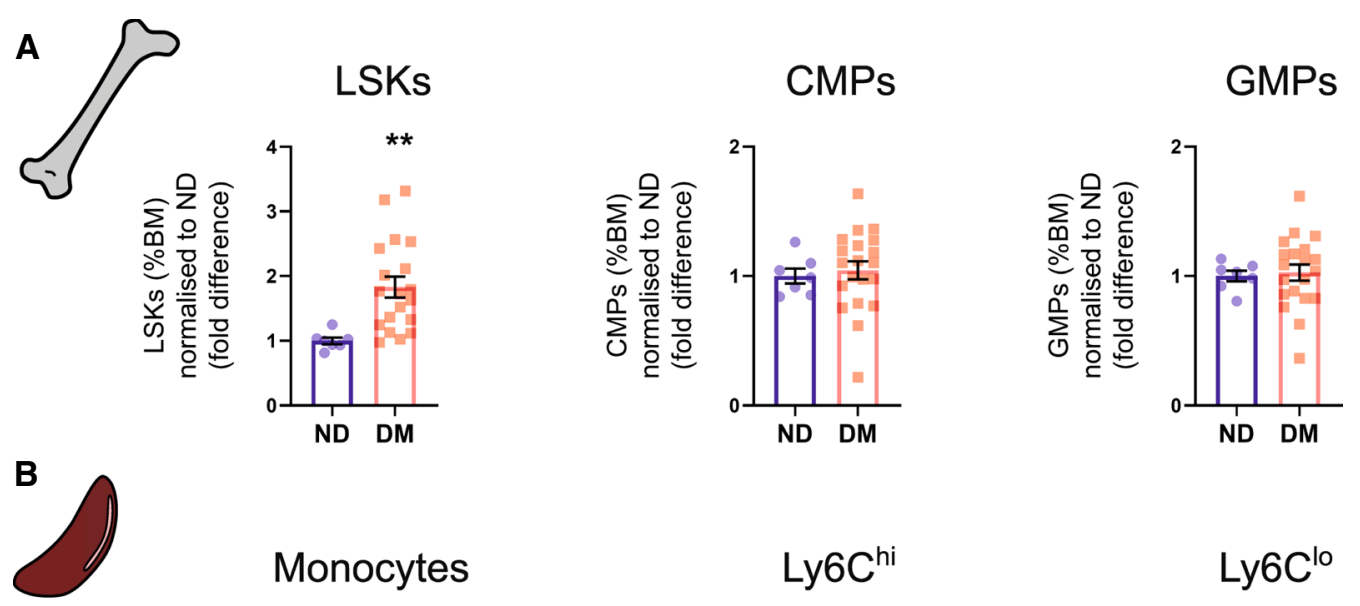

Monocytes
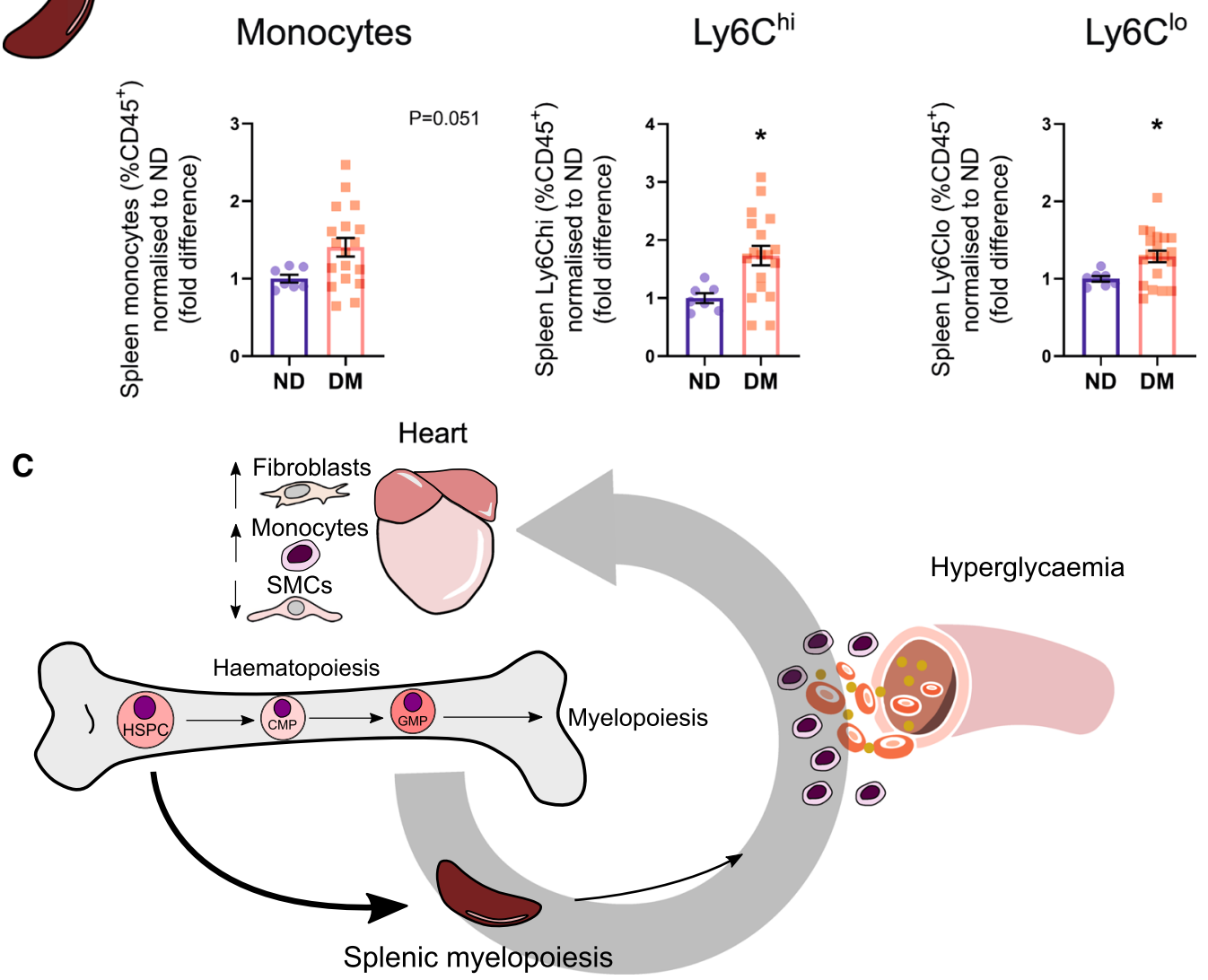

Fig. 5 Bone marrow and spleen myelopoiesis evident in mice with diabetes. A Quantified proportions of bone marrow progenitor cells. LSK cells (Lineage ${ }^{-}$, Sca- $1^{+}$, CKit $^{+}$; haematopoietic stem cells), common myeloid progenitors (CMPs) and granulocyte myeloid progenitors (GMPs). B Proportions of spleen monocytes in ND ( $n=7)$ vs. DM $(n=19)$ mice. C Proposed mechanism by which systemic monocytosis occurs in diabetic mice administered STZ-HFD. See Additional file 1: Figure $\$ 4$ and S5 for full gating strategies for flow cytometry analysis. Data displayed as mean \pm SEM. ${ }^{*} P<0.05,{ }^{*} P<0.01$ (Student's unpaired $t$-test)

abundance, to develop a framework for future mechanistic studies to consider. We revealed that with diabetesassociated diastolic dysfunction, proportions of cardiac fibroblasts are significantly increased in the myocardium. We also noted increased levels of Ly6C $\mathrm{Ch}^{\mathrm{hi}}$ monocytes and decreased levels of SMCs in diabetic hearts.
Numerous studies have implicated cardiac fibroblasts in diabetic cardiomyopathy, however their precise role in diabetes in vivo is still unknown. Cardiac fibroblasts are the primary cell type involved in deposition of extracellular matrix (ECM) in both states of acute injury or chronic stress $[14,15]$. However, in these contexts, fibroblast gene expression and phenotype are distinct [22]. 
For example, in myocardial infarction (MI), fibroblasts rapidly differentiate into activated fibroblasts and myofibroblasts-both well-established drivers of cardiac ECM deposition [23]. Conversely, we have recently reported that myofibroblasts are absent during the development of chronic fibrosis in angiotensin II-induced cardiac remodelling [14]. Observations from the present study reveal that fibroblasts are the predominant non-myocyte cell type most dramatically affected by diabetes-suggesting an important role for fibroblasts in the development of diabetes-induced HF. Indeed, chronic hyperglycaemia is known to up-regulate various pro-fibrotic genes in the diabetic heart as a whole, including Colla1, Postn, Timp2 and Ccn2 [17, 24, 25]. Furthermore, diabetes is associated with fibroblast-to-myofibroblast differentiation and ECM deposition [26, 27]. However, further research such as single-cell sequencing or targeted cell depletion experiments are needed to further elucidate the precise role of the cardiac fibroblast in diabetes and the regulatory mechanisms that drive these changes.

In the current study, we also observed an increase in monocyte numbers in the heart in diabetic mice, which is likely the result of increased systemic inflammation. In the non-injured heart, circulating leukocytes, such as monocytes primarily reside in the vascular lumens of cardiac capillaries [28], therefore reflecting changes occurring in the circulation. Systemic monocytosis is reported in both T1D and insulin resistant obese mice (i.e. leptin mutant $o b / o b$ mice and diet-induced obese mice) $[7,8]$. Consistent with systemic inflammation, the diabetic heart exhibits upregulation of pro-inflammatory cytokines such as TNF $\alpha$, MCP-1 and IL-1 $\beta$ [29-31]. Corresponding to the monocytosis, we also noted increased progenitor cells and splenic monocytes-the major site of secondary myelopoiesis [32]. Monocytosis is a wellestablished feature of diabetes and obesity/insulin resistance $[33,34]$. However, in this model we only detected a significant increase in haematopoietic stem and progenitor cells (HSPCs), but not common myeloid progenitors (CMPs) or granulocyte-macrophage progenitors (GMPs) in the bone marrow. Given HSPCs can migrate to secondary myelopoietic sites, such as the spleen, to increase monocyte numbers [35-37], our findings suggest that this may be the primary mode of monocytosis observed in our model (Fig. 5C).

In contrast to the diabetes-induced increases in cardiac fibroblasts and monocyte numbers, we observed a decrease in SMC proportions. This was unexpected given hyperglycaemia has previously been associated with inhibition of aortic vascular SMC apoptosis in T1D patients, and in STZ-induced T1D mice [38, 39]. Conversely, metabolic syndrome and hypercholesterolaemia are both associated with increased apoptosis in aortic VSMCs of mice and humans $[33,34]$. Therefore, the precise mechanism leading to the reduction in SMC proportions in the hearts of STZ-HFD mice warrants further investigation.

Although there are a number of studies examining the role of individual cardiac cell types in diabetes, to our knowledge this is the first study to consider the entire cardiac non-myocyte network to understand differences in tissue cellularity. While novel technologies such as single-cell RNA sequencing have been successfully applied to tissues such as the pancreas [35, 36, 40], kidney [41, 42] and liver [43] in diabetes, detailed interrogation of the cellular heterogeneity in these tissue systems are lacking in this context. This study invites future research to consider cellular plasticity in diabetes to better understand the development of its associated pathologies.

\section{Study limitations}

While this study provides a basis for providing new understanding of the cardiac cellular dynamics in the context of diabetes, a number of limitations are noteworthy. First, cardiomyocytes were not considered in this study, as they are too large in diameter to pass through the flow cytometer available in our laboratories. Although cardiomyocytes are detectable by histology $\left(\mathrm{PCM}^{+}{ }^{+} \mathrm{GATA}^{+}{ }^{+}\right.$cells), they are often multi-nucleated, thus counting nuclei abundance is unlikely to yield accurate information. Furthermore, we did not measure morphological changes in cardiomyocyte size or deposition of myocardial fibrosis, despite there being no differences in our previous characterisation of this model [17]. Second, we only examined male mice in our study. Given that cardiac pathology is sex-specific in mice [44, 45] and in humans [46], cardiac cellular composition and gene expression are sexually-dimorphic [12, 14]. Future work should examine the impact of biological sex in the development of diabetic cardiomyopathy. Third, the STZ-HFD model used in this study did not yield a population of mice with elevated fat mass and body weight as expected [47]. Adiposity and obesity are important comorbidities contributing to pathology in experimental and clinical type- 2 diabetes (T2D) [48, 49], albeit obesity is not essential for development of T2D [48, 49]. Importantly however, in this study mice with diabetes exhibited hyperglycaemia, impaired glucose tolerance and LV diastolic dysfunction, which are clinically relevant features of HF associated with diabetes. Furthermore, we were unable to ascertain whether the observed differences are attributed to the combination of STZ and HFD, or one of these individual insults. Future work using this model should consider the effect of STZ and HFD alone in addition to the combination of STZ-HFD to delineate the role of both factors in the development of diabetic HF. Lastly, this study did not consider how circulating populations 
of cells such as mesenchymal stem cells may contribute to the cardiac RMC compartment. Future work will consider whether resident or circulating cell populations drive the expansion of fibroblasts in the diabetic heart.

\section{Conclusion}

Here we have profiled the differences in the cardiac nonmyocyte network, observing that the cellular landscape of the heart changes in a murine model of diabetes. By quantifying proportional shifts in a wide array of cell types simultaneously, these results offer a framework for understanding the cellular mechanisms that may drive pathological remodelling of the heart during the development of diabetes-induced HF. Future research will determine the precise cellular and molecular mechanisms that drive increased fibroblast numbers and the impact of this for development of diabetic cardiomyopathy. Targeting the molecular pathways that drive these non-myocyte cellular changes may offer new therapeutic avenues to address the cardiac complications associated with diabetes.

\section{Supplementary Information}

The online version contains supplementary material available at https://doi. org/10.1186/s12933-021-01303-9.

Additional file 1: Table S1. Physiological endpoint characteristics of STZ HFD-induced murine diabetes. Table S2. Endpoint M-Mode echocardiography for assessing cardiac systolic function in murine diabetes. Table S3. Organ weights. Table S4. Flow cytometry antibody panel utilised in whole blood from mice. Table S5. Flow cytometry antibody panel utilised in myocardium from mice. Figure S1. (A) Representative images of transmitral annular blood flow via Doppler echocardiography. Quantified Doppler flow; (B) Anaesthetised heart rate (HR), (C) peak E-wave velocity, (D) A-wave velocity (E) E:A ratio, (F) deceleration time (DT) and (G) isovolumic relaxation time (IVRT). (H) Shows representative images for tissue Doppler echocardiography, quantified in figures $\mathrm{I}-\mathrm{L}$. (I) Peak $\mathrm{e}^{\prime}$ velocity $(\mathrm{P}=0.054)$, (J) peak a'velocity $(P=0.072)$, (K) e':a' ratio, $(L) E: e^{\prime}$ ratio. ND = non-diabetic, $\mathrm{DM}=$ diabetes mellitus. Data presented as mean \pm SEM and individual data points, and analysed using an unpaired t-test. Statistical significance was assumed at $P<0.05$. Figure $\mathbf{S 2}$. Flow cytometry gating strategies Heart. A) Illustrates the gating strategies used for identification of cardiac non-myocyte cell populations. Single, intact cells are first identified by the FSC-A/FSC-H gate. Next, cells are deemed 'live and metabolically active' by gating all SYTOX-Calcein + events as indicated. Live, metabolically active cells were then identified based on their cell clustering to each respective antibody (listed on the $x$ and $y$-axes). ECs = Endothelial cells, RMCs $=$ Resident mesenchymal cells, Leuks = Leukocytes, Undefined = Undefined cells, Macs = Macrophages, MHCllhi/lo = MHCllhi/lo macrophages, Fibros $=$ Fibroblasts, Mural = Mural cells, SMCs = Smooth Muscle Cells, Schwann $=$ Schwann cells, FSC-A = forward scatter area, FSC-W = forward scatter width, FSC-H = forward scatter height. B) Depicts the total number of live, metabolically active cells acquired per sample (from flow cytometry), split by treatment (ND = non-diabetic, $\mathrm{DM}=$ diabetes mellitus. Data is presented as individual values. Each line indicates the median. P $=$ NS (Student's unpaired t-test). Figure S3. Flow cytometry gating strategies Blood. Illustrates the gating strategies used for identification of circulating leukocyte populations. Single, intact cells are first identified by the FSC-A/ FSC-H gate. Live cells were identified as DAPI- (4',6-diamidino-2-phenylindole), after which cells are assigned as described in Supplementary Figure 2. FSC-A = forward scatter area, FSC-W = forward scatter width, FSC-H
= forward scatter height. Figure S4. Flow cytometry gating strategies - Bone marrow. Illustrates the gating strategies used for identification of bone marrow progenitors. Lin- = lineage negative, HSPC = haematopoietic stem cell, LSK = lineage- cKit+ cells, FSC-H = forward scatter height. Lineage cocktail = CD3, CD19, CD2, B220, TER119, CD1 1b, Gr-1, CD8, CD4. Figure S5. Flow cytometry gating strategies - Spleen. Illustrates the gating strategies used for identification of splenic monocytes. SSC-A = side scatter area. Figure S6. Histological identification of resident mesenchymal cells. Representative micrograph of murine left-ventricle stained with PCM1 and GATA4 antibodies, counter-stained with DAPI. Monochrome images (left) indicate the positive signals acquired for nuclei enumeration. Each channel is then merged and displayed in colour (right). Figure S7. Histological identification of endothelial cells. Representative micrograph of murine left-ventricle stained with DACH1 and counter-stained with DAPI. Monochrome images (left) indicate the positive signals acquired for nuclei enumeration. Both channels are then merged and displayed in colour (right). Figure S8. Chronic hyperglycaemia is evident in diabetic mice throughout study duration. Hyperglycaemia was first detected 2-weeks after the commencement of STZ-HFD administration, and remains elevated until endpoint (measured fortnightly). Data is presented as mean \pm SEM. Statistical significance was determined by a repeated measures ANOVA using a Tukey's multiple comparison post-hoc test. ${ }^{* * P}<0.01$, ${ }^{* * * P}$ $<0.001,{ }^{* * * *} P<0.0001$

\section{Acknowledgements}

We wish to acknowledge the Monash Micro-Imaging (MMI) facility for provision of instrumentation and training.

\section{Authors' contributions}

CDC, MJD, RHR, ARP, conceived the experiments, GD, MJD, AJM and MKSL provided intellectual input. CDC, ARP, MJD, GEF, CK, TLG, MCF, DP, MD performed experiments. HK, DD performed echocardiography imaging and assisted with quality control of echocardiography analysis. CDC wrote the manuscript under ARP and RHR's guidance. RHR, ARP, MJD, GD edited the manuscript. All co-authors read and approved the final manuscript.

\section{Funding}

CDC, CK and TLG are supported by the La Trobe University Postgraduate Research Scholarship (LTUPRS), Research Training Program Fees Off-set (RTPFo) Scholarship. CDC is supported by a Baker Institute 'Bright Sparks' Scholarship. TLG is supported by Defence Science Institute (DSI) RhD Grant. This work was supported by a project grant to RHR and MJD from the National Health and Medical Research Council (NHMRC) of Australia (APP1158013), and an NHMRC fellowship to RHR (APP1059960) and in part by an infrastructure grant from the Victorian Government of Australia. This work was also supported by an NHMRC Ideas Grant (GNT1188503) to ARP.

\section{Availability of data and materials}

The datasets used and/or analysed during the current study are available from the corresponding author on reasonable request.

\section{Declarations}

\section{Ethics approval}

All animal-related experiments were approved by the Alfred Research Alliance (ARA) Animal Ethics Committee (Ethics number: E/1681/2016/B) and were in accordance with NHMRC guidelines.

\section{Consent for publication}

Not applicable.

\section{Competing interests}

None.

\section{Author details}

${ }^{1}$ Heart Failure Pharmacology, Drug Discovery Biology, Monash Institute of Pharmaceutical Sciences, 399 Royal Parade, Parkville, VIC 3052, Australia. ${ }^{2}$ Cardiac Cellular Systems, Baker Heart and Diabetes Institute, 75 Commercial 
Road, Melbourne, VIC 3004, Australia. ${ }^{3}$ Haematopoiesis and Leukocyte Biology, Baker Heart and Diabetes Institute, Prahran, VIC, Australia. ${ }^{4}$ Preclinical Cardiology, Microsurgery \& Imaging Platform, Baker Heart and Diabetes Institute, Prahran, VIC, Australia. ${ }^{5}$ Department of Physiology, Microbiology and Anatomy, La Trobe University, Bundoora, VIC, Australia. ${ }^{6}$ Baker Department of Cardiometabolic Health, The University of Melbourne, Parkville, VIC 3010, Australia.

Received: 18 March 2021 Accepted: 19 May 2021

Published online: 01 June 2021

\section{References}

1. Raghavan S, Vassy JL, Ho YL, Song RJ, Gagnon DR, Cho K, et al. Diabetes mellitus-related all-cause and cardiovascular mortality in a national cohort of adults. J Am Heart Assoc. 2019. https://doi.org/10.1161/JAHA. 118.011295.

2. Ng ACT, Delgado V, Borlaug BA, Bax JJ. Diabesity: the combined burden of obesity and diabetes on heart disease and the role of imaging. Nat Rev Cardiol. 2020;18(4):291-304.

3. Ogurtsova K, da Rocha Fernandes JD, Huang Y, Linnenkamp U, Guariguata L, Cho NH, et al. IDF Diabetes Atlas: Global estimates for the prevalence of diabetes for 2015 and 2040. Diabetes Res Clin Pract. 2017:128:40-50.

4. Petar M, Mark C, Gerasimos S, Stefan D, Walter J, Boer D, et al. Type 2 diabetes mellitus and heart failure: a position statement from the Heart Failure Association of the European Society of Cardiology Type 2 diabetes mellitus and heart failure: a position statement from the Heart Failure Association of the European. Eur J Heart Failure. 2018;20:853-72.

5. Rubler S, Dlugash J, Yuceoglu YZ, Kumral T, Branwood AW, Grishman A. New type of cardiomyopathy associated with diabetic glomerulosclerosis. Am J Cardiol. 1972;30:595-602.

6. Ritchie RH, Dale AE. Basic mechanisms of diabetic heart disease. Circ Res. 2020;126:1501-25.

7. Nagareddy PR, Murphy AJ, Stirzaker RA, Hu Y, Yu S, Miller RG, et al. Hyperglycemia promotes myelopoiesis and impairs the resolution of atherosclerosis. Cell Metab. 2013;17:695-708.

8. Nagareddy PR, Kraakman M, Masters SL, Stirzaker RA, Gorman DJ, Grant RW, et al. Adipose tissue macrophages promote myelopoiesis and monocytosis in obesity. Cell Metab. 2014;19:821-35.

9. Wong SL, Demers M, Martinod K, Gallant M, Wang Y, Goldfine AB, et al. Diabetes primes neutrophils to undergo NETosis, which impairs wound healing. Nat Med. 2015;21:815-9.

10. Pinto AR, llinykh A, Ivey MJ, Kuwabara JT, D'Antoni ML, Debuque R, et al. Revisiting cardiac cellular composition. Circ Res. 2016;118:400-9.

11. Skelly DA, Squiers GT, Mclellan MA, Bolisetty MT, Robson P, Rosenthal $N A$, et al. Single-cell transcriptional profiling reveals cellular diversity and intercommunication in the mouse heart. Cell Rep. 2018:22:600-10.

12. Squiers GT, Mclellan MA, llinykh A, Branca J, Rosenthal NA, Pinto AR. Cardiac cellularity is dependent upon biological sex and is regulated by gonadal hormones. Cardiovasc Res. 2020;cva265:1-25.

13. Litviňuková M, Talavera-López C, Maatz H, Reichart D, Worth CL, Lindberg EL, et al. Cells of the adult human heart. Nature. 2020;588:466-72.

14. McLellan MA, Skelly DA, Dona MSI, Squiers GT, Farrugia GE, Gaynor TL, et al. High-resolution transcriptomic profiling of the heart during chronic stress reveals cellular drivers of cardiac fibrosis and hypertrophy. Circulation. 2020;142:1448-63.

15. Forte E, Skelly DA, Chen M, Daigle S, Morelli KA, Hon O, et al. Dynamic interstitial cell response during myocardial infarction predicts resilience to rupture in genetically diverse mice. Cell Rep. 2020;30:3149-3163.e6.

16. Farbehi N, Patrick R, Dorison A, Xaymardan M, Janbandhu V, Wystub-Lis $K$, et al. Single-cell expression profiling reveals dynamic flux of cardiac stromal, vascular and immune cells in health and injury. Elife. 2019;8:1-39.

17. Tate M, Prakoso D, Willis AM, Peng C, Deo M, Qin CX, et al. Characterising an alternative murine model of diabetic cardiomyopathy. Front Physiol. 2019;10:1-15.

18. Tate M, Higgins GC, de Blasio MJ, Lindblom R, Prakoso D, Deo M, et al. The mitochondria-targeted methylglyoxal sesquestering compound, MitoGamide, is cardioprotective in the diabetic heart. Cardiovasc Drugs Ther. 2019;33:669-74.
19. Westermann D, Rutschow S, Jäger S, Linderer A, Anker S, Riad A, et al. Contributions of inflammation and cardiac matrix metalloproteinase activity to cardiac failure in diabetic cardiomyopathy: The role of angiotensin type 1 receptor antagonism. Diabetes. 2007;56:641-6.

20. Chu PY, Walder K, Horlock D, Williams D, Nelson E, Byrne M, et al. CXCR4 antagonism attenuates the development of diabetic cardiac fibrosis. PLOS ONE. 2015;10:1-13.

21. Huynh K, Bernardo BC, McMullen JR, Ritchie RH. Diabetic cardiomyopathy: Mechanisms and new treatment strategies targeting antioxidant signaling pathways. Pharmacol Ther. 2014;142:375-415.

22. Tallquist MD, Molkentin JD. Redefining the identity of cardiac fibroblasts. Nat Rev Cardiol. 2017;14:484-91.

23. Krstevski C, Cohen CD, Dona MSI, Pinto AR. New perspectives of the cardiac cellular landscape: mapping cellular mediators of cardiac fibrosis using single-cell transcriptomics. Biochem Soc Trans. 2020;48:2483-93.

24. Hutchinson KR, Lord CK, West TA, Stewart JA. Cardiac fibroblast-dependent extracellular matrix accumulation is associated with diastolic stiffness in type-2 diabetes. PLoS ONE. 2013;8:e72080.

25. Prakoso D, de Blasio M, Kiriazis H, Qian H, Deo M, Jap E, et al. Cardiac remodelling and inflammation associated with diabetic cardiomyopathy is mitigated by phosphoinositide 3-kinase P110a gene delivery (abstract). Heart Lung Circ. 2017;26:S50. https://doi.org/10.1016/j.hlc.2017.06.016.

26. Fowlkes V, Clark J, Fix C, Law BA, Morales MO, Qiao X, et al. Type II diabetes promotes a myofibroblast phenotype in cardiac fibroblasts. Life Sci. 2013;92:669-76.

27. Sedgwick B, Riches K, Bageghni SA, O'Regan DJ, Porter KE, Turner NA. Investigating inherent functional differences between human cardiac fibroblasts cultured from nondiabetic and Type 2 diabetic donors. Cardiovasc Pathol. 2014;23:204-10.

28. Epelman S, Lavine KJ, Randolph GJ. Origin and functions of tissue macrophages. Immunity. 2014:41:21-35.

29. Jadhav A, Tiwari S, Lee P, Ndisang JF. The heme oxygenase system selectively enhances the anti-inflammatory macrophage-M2 phenotype, reduces pericardial adiposity, and ameliorated cardiac injury in diabetic cardiomyopathy in Zucker diabetic fatty rats. J Pharmacol Exp Ther. 2013;345:239-49.

30. Qin CX, Sleaby R, Davidoff AJ, Bell JR, de Blasio MJ, Delbridge LM, et al. Insights into the role of maladaptive hexosamine biosynthesis and O-GlcNAcylation in development of diabetic cardiac complications. Pharmacol Res. 2017;116:45-56. https://doi.org/10.1016/j.phrs.2016.12.016.

31. Kassan M, Choi SK, Galán M, Bishop A, Umezawa K, Trebak M, et al. Enhanced NF-KB activity impairs vascular function through PARP-1-, SP-1-, and COX-2-dependent mechanisms in type 2 diabetes. Diabetes. 2013;62:2078-87.

32. Swirski FK, Nahrendorf M. Leukocyte behavior in atherosclerosis, myocardial infarction, and heart failure. Science. 2013;339:161-6.

33. Clarke MCH, Figg N, Maguire JJ, Davenport AP, Goddard M, Littlewood $\mathrm{TD}$, et al. Apoptosis of vascular smooth muscle cells induces features of plaque vulnerability in atherosclerosis. Nat Med. 2006;12:1075-80.

34. Martínez-Hervás S, Vinué Á, Núñez L, Andrés-Blasco I, Piqueras L, TomásReal J, et al. Insulin resistance aggravates atherosclerosis by reducing vascular smooth muscle cell survival and increasing CX3CL1/CX3CR1 axis. Cardiovasc Res. 2014;103:324-36.

35. Segerstolpe Å, Palasantza A, Eliasson P, Andersson EM, Andréasson AC, Sun $X$, et al. Single-cell transcriptome profiling of human pancreatic islets in health and type 2 diabetes. Cell Metab. 2016;24:593-607.

36. Wang YJ, Schug J, Won KJ, Liu C, Naji A, Avrahami D, et al. Singlecell transcriptomics of the human endocrine pancreas. Diabetes. 2016;65:3028-38.

37. Rai V, Quang DX, Erdos MR, Cusanovich DA, Daza RM, Narisu N, et al. Single-cell ATAC-Seq in human pancreatic islets and deep learning upscaling of rare cells reveals cell-specific type 2 diabetes regulatory signatures. Mol Metab. 2020;32:109-21.

38. Ruiz E, Gordillo-Moscoso A, Padilla E, Redondo S, Rodriguez E, Reguillo $F$, et al. Human vascular smooth muscle cells from diabetic patients are resistant to induced apoptosis due to high $\mathrm{BCl}-2$ expression. Diabetes. 2006;55:1243-51.

39. Hall JL, Matter CM, Wang X, Gibbons GH. Hyperglycemia inhibits vascular smooth muscle cell apoptosis through a protein kinase C-dependent pathway. Circ Res. 2000;87:574-80. 
40. Veres A, Baron M, Shen-Orr SS, Klein AM, Melton DA, Faust AL, et al. A single-cell transcriptomic map of the human and mouse pancreas reveals inter- and intra-cell population structure. Cell Syst. 2016;3:346-360. e4. https://doi.org/10.1016/j.cels.2016.08.011.

41. Fu J, Akat KM, Sun Z, Zhang W, Schlondorff D, Liu Z, et al. Single-cell RNA profiling of glomerular cells shows dynamic changes in experimental diabetic kidney disease. J Am Soc Nephrol. 2019;30:533-45.

42. Wilson PC, Wu H, Kirita Y, Uchimura K, Ledru N, Rennke HG, et al. The single-cell transcriptomic landscape of early human diabetic nephropathy. Proc Natl Acad Sci USA. 2019;116:19619-25.

43. Xiong $X$, Kuang H, Ansari S, Liu T, Gong J, Wang S, et al. Landscape of intercellular crosstalk in healthy and NASH liver revealed by single-cell secretome gene analysis. Mol Cell. 2019;75:644-660.e5.

44. Alex L, Russo I, Holoborodko V, Frangogiannis NG. Characterization of a mouse model of obesity-related fibrotic cardiomyopathy that recapitulates features of human heart failure with preserved ejection fraction. Am J Physiol Heart Circ Physiol. 2018;315:H934-49.

45. Kessler EL, Rivaud MR, Vos MA, van Veen TAB. Sex-specific influence on cardiac structural remodeling and therapy in cardiovascular disease. Biol Sex Differ. 2019. https://doi.org/10.1186/s13293-019-0223-0.
46. Beale AL, Meyer PMD, MarwickTH, Lam CSP, Kaye DM. Sex differences in cardiovascular pathophysiology why women are overrepresented in heart failure with preserved ejection fraction. Circulation. 2018;138:198-205.

47. Kahn SE, Cooper ME, del Prato S. Pathophysiology and treatment of type 2 diabetes: perspectives on the past, present, and future. Lancet. 2014;383:1068-83.

48. Yoon K-H, Lee J-H, Kim J-W, Cho JH, Choi Y-H, Ko S-H, et al. Epidemic obesity and type 2 diabetes in Asia. Lancet. 2006:368:1681-8.

49. George AM, Jacob AG, Fogelfeld L. Lean diabetes mellitus: an emerging entity in the era of obesity. World J Diabetes. 2015;6:613.

\section{Publisher's Note}

Springer Nature remains neutral with regard to jurisdictional claims in published maps and institutional affiliations.
Ready to submit your research? Choose BMC and benefit from:

- fast, convenient online submission

- thorough peer review by experienced researchers in your field

- rapid publication on acceptance

- support for research data, including large and complex data types

- gold Open Access which fosters wider collaboration and increased citations

- maximum visibility for your research: over $100 \mathrm{M}$ website views per year

At BMC, research is always in progress.

Learn more biomedcentral.com/submissions 